\title{
IMPULSE NOISE REMOVAL FROM MEDICAL IMAGES USING FUZZY GENETIC ALGORITHM
}

\author{
Anisha K K and Dr.M.Wilscy \\ Department of Computer Science, University of Kerala, Kariavattom, \\ Thiruvananthapuram 695 581, Kerala, India \\ anisha.kelamkumarath@gmail.com, wilsyphilipose@hotmail.com
}

\begin{abstract}
Medical images are analyzed for diagnosis of various diseases. But, they are susceptible to impulse noise. Noise removal can be done much more efficiently by a combination of image filters or a composite filter, than by a single image filter. Determining the appropriate filter combination is a difficult task. In this paper, we propose a technique that uses Fuzzy Genetic Algorithm to find the optimal composite filters for removing all types of impulse noise from medical images. Here, a Fuzzy Rule Base is used to adaptively change the crossover probability of the Genetic Algorithm used to determine the optimal composite filters. The results of simulations performed on a set of standard test images for a wide range of noise corruption levels shows that the proposed method outperforms standard procedures for impulse noise removal both visually and in terms of performance measures such as PSNR, IQI and Tenengrad values.
\end{abstract}

\section{KEYWORDS}

Adaptive Genetic Algorithm, Fuzzy Genetic Algorithm (FGA), Fuzzy Rule Base (FRB), Genetic Algorithm (GA), Image filters, Impulse noise

\section{INTRODUCTION}

Digital image processing plays a key role in medical diagnosis. Medical images are obtained and analyzed to determine the presence or absence of abnormalities such as tumor, which is vital in understanding the type and magnitude of a disease. Unfortunately, medical images are susceptible to impulse noise during acquisition, storage and transmission. Hence, image denoising is a primary precursor for medical image analysis tasks. Conventional smoothening filters and median filters are the most popular filters for noise reduction in digital images [1].But, a single smoothening or median filter is not enough for completely removing the noise, especially when the noise level is high. Also, it may not preserve image details such as edges during filtering. This is a serious issue in medical image analysis because loss of image details results in inaccurate image analysis which may prove fatal to the life of a person. Hence, many methods have been proposed for noise removal from medical images. While some of these methods use complicated formulations, others require deep knowledge about image noise factors. Hence, a simple noise reduction method that removes noise well and preserves image details without relying on image noise factors is desirable.

Applying a set of denoising and enhancement filters successively on a noisy image may remove noise and preserve image details much more efficiently than a single median or smoothening filter. Such a set of standard filters is called a composite filter. The type of the filters in the composite filter, as well as the order in which the filters are to be applied must be appropriately chosen for good results. Jin Hyuk Hong, Sung Bae Cho and Ung Keun Cho proposed a method 
The International Journal of Multimedia \& Its Applications (IJMA) Vol.3, No.4, November 2011

that uses Genetic Algorithm (GA) [2] to determine composite filters that remove different levels of impulse noise from an image [3].They have extended this method to determine composite filters that performs local and global image enhancement [4]. In these methods, the GA considers a set of possible filter combinations of a particular length, selects the best combinations among them according to a fitness value assigned to each combination based on a fitness function, and applies genetic operators such as crossover and mutation [2] on the selected combinations to create the next generation of composite filters. This process is repeated, enabling GA to find the optimal composite filters. In the work in [3] and [4], GA parameters, which affect the quality of the solutions produced, are kept fixed. If these parameters are not assigned with suitable values, GA may converge to a sub optimal solution, or it may take a long time to converge to the optimal solution. However, choosing the best parameter values is difficult because the parameter values are problem dependent.

The performance of GA can be improved by adaptively varying its parameters instead of keeping them fixed. Fuzzy logic [7] based techniques have been used for adaptively selecting GA parameters [5] [8-10]. In Fuzzy Genetic Algorithm (FGA) [5], [8-10], a Fuzzy Rule Base (FRB) [7] is used to adaptively vary any of the GA parameters.

The proposed method uses an FGA [5] to determine the optimal filters that can remove different levels of impulse noise from medical images. From a pool of standard filters, the GA part of FGA selects several filters and constructs a composite filter. GA analyses such a set of composite filters and determine the optimal filters for removing different levels of impulse noise. The crossover probability [2] of GA is adapted by the fuzzy logic part of FGA, where a Fuzzy Rule Base (FRB) is used to determine the amount of variation that should be undergone by the crossover probability value in order to improve the quality of the solutions produced. The proposed method does not rely on deep knowledge about the type of image noise factors. Hence, it can be used to remove almost all types of impulse noise [11] from images.

The proposed method has been tested on medical images and its performance has been evaluated both subjectively as well as objectively using performance metrics such as Peak Signal to Noise Ratio (PSNR) value, Tenengrad measure and Image Quality Index (IQI) [6] value. These evaluations clearly show the superiority of the proposed method over standard procedures for impulse noise removal from medical images.

The rest of the paper is organized as follows. Section 2 explains the different types of impulse noise. Section 3 gives a detailed account of the proposed method, where the design and working of the FGA is explained. Section 4 discusses the experimental results. Section 5 provides conclusions.

\section{IMPULSE NOISE MODELS}

There are four different types or models of impulse noise [11]. They are as follows:

Noise Model 1: This noise is also known as salt-and-pepper impulse noise. Here, pixels are randomly corrupted by two fixed extreme values, 0 and 255 (for gray level image), generated with the same probability. That is, if $\mathrm{P}$ is the noise density, then the noise density of salt (P1) and pepper $(\mathrm{P} 2)$ is $\mathrm{P} / 2$.

Noise Model 2: This type of noise is similar to Noise Model 1, but here, each pixel might be corrupted by either pepper noise or salt noise with unequal probabilities. That is $\mathrm{P} 1 \neq \mathrm{P} 2$.

Noise Model 3: Instead of two fixed values, impulse noise could be more realistically modeled by two fixed ranges that appear at both ends with a length of $\mathrm{m}$ each, respectively. That is, $[0, \mathrm{~m}]$ 
The International Journal of Multimedia \& Its Applications (IJMA) Vol.3, No.4, November 2011

denotes salt and [255-m, 255] denotes pepper. Here for noise density P, P1=P2= P/2. This noise is also known as random impulse noise or uniform noise.

Noise Model 4: This noise is similar to Noise Model 3 but here probability densities of low intensity impulse noise and high intensity impulse noise are different. That is, $\mathrm{P} 1 \neq \mathrm{P} 2$.

Many techniques have been proposed for impulse noise removal from grayscale images. Some of these methods work only for either low density noisy images or high density noisy images. Some other techniques are specifically designed for certain noise models. Some techniques use complicated formulations or require deep knowledge about the image noise factors. The proposed method, which is explained in section 3, is a method which removes any level of impulse noise, is applicable for almost all noise models, does not use complicated formulations and does not require deep knowledge on image noise factors.

\section{The Proposed Method using Fuzzy Genetic Algorithm}

The proposed method for impulse noise removal consists of two parts: A GA part and a Fuzzy Logic part. The GA part selects several filters and constructs a composite filter. GA analyses such a set of composite filters and determine the optimal filters for removing different levels of impulse noise. The crossover probability [2] of GA, which determines the number of selected solutions that undergo crossover operation, is adapted by the fuzzy logic part of FGA, where an FRB is used to determine the amount of variation that should be undergone by the crossover probability value in order to improve the quality of the solutions produced. The following subsections explain these two parts of the proposed method.

\subsection{The GA Part}

When there are $\mathbf{m}$ filters in the filter pool, optimal composite filters containing $\mathbf{l}$ standard filters are to be determined from a total of $(\mathbf{m}+\mathbf{1})^{\mathbf{1}}$ filter combinations, where $\mathbf{m}+\mathbf{1}$ includes the case of not using any filter on the image. Trying all cases to find out the best one is practically impossible, especially when $\mathbf{m}$ is large. In this paper, GA is used to find the optimal composite filters. In GA [2], each solution to the problem to be solved is called an individual or a chromosome. GA starts by randomly initializing a set or a population of individuals. This is the first generation of individuals. Each individual is assigned a fitness value based on a fitness function. GA selects those individuals with a good fitness value and applies operations such as crossover and mutation on them to create the next generation of individuals. This process is repeated until GA satisfies a predefined termination criterion such as the number of generations created, upon which GA is expected to have produced very good individuals.

Table 1 shows the filter pool used in this paper which contains 23 image filters, each indexed by a value from $1-23$. Value 0 represents the case where no filtering operation is performed. The first 3 filters are histogram brightness measures that adjust the value of the pixel $p$ in the image according to (1) for a given scale $(-100<=\mathrm{s}<=100)$.

$$
v_{\text {new }(p)}=v_{\text {old }}(p)+\frac{v_{\text {old }}(p) * s}{100}
$$

Filters $4-7$ are histogram contrast measures that adjusts the value of the pixel $\mathrm{p}$ for a given scale $(-127<=$ s $<=127)$ as shown in (2).

$$
v_{\text {new }}(p)=v_{\text {old }}(p)+\frac{v_{\text {old }}(p)-128 * s}{128}
$$

Filter 8 performs contrast stretching by spanning the range of intensity values in an image, [c, d], to a desired range of values $[a, b]$. It scales each image pixel $p$ according to (3). 
The International Journal of Multimedia \& Its Applications (IJMA) Vol.3, No.4, November 2011

$$
v_{\text {new }}(p)=v_{\text {old }}(p)-c *\left(\frac{b-a}{d-c}\right)+a
$$

Filter 9equalizes the histogram of the image, thereby improving the image contrast. Filters $10-$ 13 are edge enhancement filters of different types. Filters $14-22$ are standard median filters of different sizes and shapes. Filter 23 is an adaptive median filter with a maximum window size of $7[1]$.

Table 1. Description of image filters used in this paper.

\begin{tabular}{|c|c|c|}
\hline Filter & Type & Index \\
\hline Brightness & 3 values of $\mathrm{s}$ & $1 \sim 3$ \\
\hline Contrast & 4 values of $\mathrm{s}$ & $4 \sim 7$ \\
\hline Stretch & - & 8 \\
\hline Equalize & - & 9 \\
\hline Sharpening & 4 masks & $10 \sim 13$ \\
\hline $\begin{array}{c}\text { Median, } \\
\text { Adaptive Median }\end{array}$ & 10 masks & $14 \sim 23$ \\
\hline None & & 0 \\
\hline
\end{tabular}

Each composite filter is represented by a string of $\mathbf{l}$ integers, where each integer is the index of the corresponding filter in the composite filter and $\mathbf{l}$ is the number of standard filters in the composite filter.

At first, GA randomly initializes a population of composite filters. Then, the fitness of each composite filter is evaluated using the fitness function given in (4). Here, the objective of GA is to find the optimal composite filter that can remove impulse noise from all the training images in a given training set. The training images are created by artificially corrupting an image with different levels of impulse noise. In (4), $n$ is the number of training images used, $\mathrm{MAE}_{\mathrm{i}}$ is the mean absolute error (MAE) of the output image obtained after applying the composite filter $\mathrm{x}$ on the $i^{\text {th }}$ training image, and $\mathrm{MAE}_{\max }$ is the maximum MAE; it would be 255 for 8-bit grayscale images. The fitness value $f(x)$, which is the average of the performance of $x$ on all the training images, is assigned to $\mathrm{x}$. From (4), it is clear that the composite filter $\mathrm{x}$ receives a high fitness value if it can considerably remove the noise from all the training images.

$$
\mathrm{f}(\mathbf{x})=\frac{1}{n} \sum_{i=1}^{n} 1-\left(M A E_{i} / M A E_{\text {max }}\right)
$$

GA then selects the composite filters with high fitness value from the current generation using Roulette Wheel selection strategy [2], where selection is based on the probability assigned to each composite filter proportional to its fitness value. Then, genetic operators such as crossover and mutation are applied on the selected individuals, to produce the next generation of individuals. Elitist-strategy [2] that passes the best individuals of the current generation directly to the next generation is also used here. This process is repeated until a predefined termination criterion is satisfied. Here, the termination criterion is the maximum number of generations created by GA. Since the GA selects only the best solutions from each generation and as Elitism is used, after a particular number of generations, the population contains only good composite filters, from which the optimal composite filters for removing different levels of impulse noise will be the ones with the highest fitness values. 
The International Journal of Multimedia \& Its Applications (IJMA) Vol.3, No.4, November 2011

When GA parameter values are kept fixed for solving a problem, it must be ensured that the parameter values suit the problem. Otherwise, the convergence of GA may be to a sub optimal solution. Even if GA converges to the optimal solution, it may take a large amount of time to do so. The occurrence of these problems can be avoided by adaptively varying the GA parameters. In the proposed method, the fuzzy logic part adapts the crossover probability of the GA using a FRB. The fuzzy logic part is explained in detail in the next subsection.

\subsection{The Fuzzy Logic Part}

An FGA [5], [8 - 10] is an adaptive GA in which a Fuzzy Rule Base (FRB) is used to adapt one or more of the GA parameters so as to increase the quality of the solutions produced by GA. FGA accepts one or more values that indicate the quality of the outputs produced by GA as inputs. These values are fuzzified[7] using the corresponding membership functions[7]. From these fuzzified input values, one or more fuzzy outputs are determined using a FRB [7]. These outputs are then defuzzified [7] using the output membership functions. The defuzzified outputs enable the adaptive variation of one or more GA parameters, thereby enabling GA to converge to the most optimal solution. It also results in an increase in the speed of convergence of the GA to the best solution.

In the proposed method, a FRB is used to adapt the crossover probability of GA. Probability of crossover $p_{c}$ determines the number of individuals in the population that must undergo crossover operation. A high crossover probability value results in loss of good chromosomes in the current generation, and a low crossover probability value results in loss of diversity in the subsequent generations because these generations may get constituted of copies of just a few average individuals. Both these cases may result in convergence of GA to a suboptimal solution. Hence it is important to have an optimal crossover probability value.

The fuzzy logic part accepts Genotypic diversity (GD) and Phenotypic diversity (PD) as inputs [5].GD and PD are two measures that depict the quality of the composite filters produced by GA.

GD represents the genetic diversity of the population and it is evaluated as shown in (5).

$$
\mathrm{GD}=\left(\mathrm{d}-\mathrm{d}_{\min }\right) /\left(\mathrm{d}_{\max }-\mathrm{d}_{\min }\right)
$$

Where $\mathrm{d}, \mathrm{d}_{\max }$ and $\mathrm{d}_{\min }$ are the average, maximum and minimum distances of the chromosomes in the population from the composite filter with the highest fitness value.

$\mathrm{PD}$, as shown in (6), is the ratio of average fitness of the population, $\mathrm{f}_{\text {avg }}$, to the best fitness $\mathrm{f}_{\text {best }}$.

$$
\mathrm{PD}=\mathrm{f}_{\text {avg }} / \mathrm{f}_{\text {best }}
$$

Figures 1(a) and (b) shows the membership functions of GD and PD respectively which are used for fuzzifying GD and PD values.
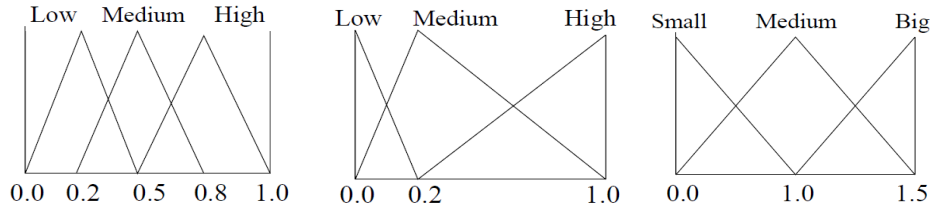

Figure 1. Membership function of (a) GD (b) PD (c) $\delta p_{c}$ 
The International Journal of Multimedia \& Its Applications (IJMA) Vol.3, No.4, November 2011

Table 2 shows the FRB which is used to determine the fuzzy output value, which is defuzzified using the membership function shown in figure 1(c) to obtain the crisp output value $\delta \mathrm{p}_{\mathrm{c}}$. GD and PD values range from Low to High, for which the change in $\delta p_{c}$, which ranges from Small to Big, is given in the respective cells. When GD and PD values are 'Low', the population is diverse, even if it has not converged to the best solution. In this case, a low crossover probability is desired to prevent loss of this diversity due to crossover. Hence, $\delta p_{c}$ is given a 'Small' value, to allow as little crossover operations as possible. Similar arguments follow for all the conditions specified in the rule base.

$\delta p_{c}$, which ranges from $[0,1.5]$, determines the degree to which the current $p_{c}$ value, which is kept within the range $[0.25,0.75]$, should vary. The new $p_{c}$ value is obtained by multiplying the $\delta p_{c}$ value with the current $\mathrm{p}_{\mathrm{c}}$ value.

Table 2. Description of image filters used in this paper.

\begin{tabular}{|c|c|c|c|}
\hline \multirow{2}{*}{ GD } & \multicolumn{3}{|c|}{ PD } \\
\cline { 2 - 4 } & Low & Medium & High \\
\hline Low & Small & Small & Medium \\
\hline Medium & Big & Big & Medium \\
\hline High & Big & Big & Medium \\
\hline
\end{tabular}

In the proposed method, GA creates the first generation of composite filters using a randomly initialized $p_{c}$ value. The GD and PD values for this generation are fed into the fuzzy logic part of FGA, which calculates the value of $\delta p_{c}$, which is multiplied with the current $p_{c}$ value. This adapted $p_{c}$ value is given to the GA, which uses it to create the next generation to produce a better population of composite filters. The entire process is repeated until GA satisfies its termination criterion.

\section{RESUlTS AND DISCUSSION}

The proposed method was implemented and tested on medical images. The experimental setup is given below:

Images used: 256 x 194 MRI (Magnetic Resonance Imaging) brain image, 200 x 200 MRI knee image, 350 x 250 mammogram image and 185 x 192 MRI head image (Figure 2).

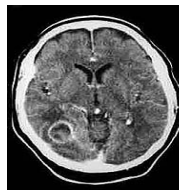

(a)

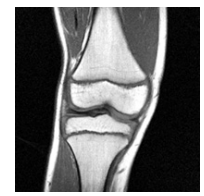

(b)

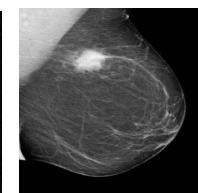

(c)

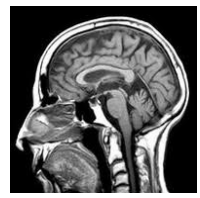

(d)

Figure 2. (a) Brain. (b) Knee. (c) Mammogram. (d) Head.

Training images:20 noisy images created by artificially corrupting brain, knee, mammogram and head images by model 1 , model 2 , model 3 (with interval $=6$ ) and model 4 (with interval $=4$ ) impulse noise factors respectively with corruption rates of $10 \%, 30 \%, 50 \%, 70 \%$ and $90 \%$.

Test images: Brain, knee, mammogram and head images corrupted with various levels of impulse noise. 
The International Journal of Multimedia \& Its Applications (IJMA) Vol.3, No.4, November 2011

GA parameters: After conducting several trials, the following values are found to be optimal for the different GA parameters:

Population size $=50$

Number of generations created by GA $=250$

The length of the composite filter obtained for each training image is given below:

- Brain image with noise model 1

$\begin{array}{ll}\circ & 10 \% \text { noise }-2 \\ \circ & 30 \% \text { noise }-2 \\ \circ & 50 \% \text { noise }-3 \\ \circ & 70 \% \text { noise }-5 \\ \circ & 90 \% \text { noise }-5\end{array}$

- Knee image with noise model 2
○ $10 \%$ noise -2
○ $30 \%$ noise -3
○ $50 \%$ noise -5
- $70 \%$ noise -5
○ $90 \%$ noise -5

- Mammogram image with noise model 3 and with interval $=6$

$$
\begin{array}{cl}
\circ & 10 \% \text { noise }-2 \\
\circ & 30 \% \text { noise }-2 \\
\circ & 50 \% \text { noise }-3 \\
\circ & 70 \% \text { noise }-5 \\
\circ & 90 \% \text { noise }-5
\end{array}
$$

- Head image with noise model 4 and with interval $=4$

$$
\begin{array}{ll}
\circ & 10 \% \text { noise }-2 \\
\circ & 30 \% \text { noise }-2 \\
\circ & 50 \% \text { noise }-5 \\
\circ & 70 \% \text { noise }-5 \\
\circ & 90 \% \text { noise }-5
\end{array}
$$

Mutation probability $=0.05$.

The initial crossover probability value used is 0.7 . The final crossover probability value obtained after 250 generations is 0.75 .

Table 3 shows the composite filters created by the proposed method for the training images. Here, it can be seen that as the amount of noise in the image increases, the length and the complexity of the composite filter also increases, which shows that the proposed method adaptively creates composite filters for different noise levels. The composite filters evolved for the brain, knee, mammogram and head images are almost different for the same noise levels. This shows that the composite filter evolution depends on the characteristic features of the training images.

Table 3. Composite filters evolved by FGA for the training images

\begin{tabular}{|c|c|c|c|}
\hline $\begin{array}{l}\text { Noise } \\
\text { Model }\end{array}$ & Image & $\begin{array}{c}\text { Impulse } \\
\text { Noise } \\
\text { Level } \\
(\%)\end{array}$ & $\begin{array}{c}\text { Composite filters produced by FGA (in the order } \\
\text { of application) }\end{array}$ \\
\hline \multirow{2}{*}{1} & Brain & $\mathbf{1 0}$ & Brightness value control with $\mathrm{s}=0$, Adaptive Median \\
\cline { 3 - 4 } & & $\mathbf{3 0}$ & Brightness value control with $\mathrm{s}=0$, Adaptive Median \\
\cline { 3 - 4 } & $\mathbf{5 0}$ & $\begin{array}{c}\text { Brightness value control with } \mathrm{s}=0 \text {, Adaptive Median, } \\
3 \times 1 \text { vertical median }\end{array}$ \\
\hline
\end{tabular}


The International Journal of Multimedia \& Its Applications (IJMA) Vol.3, No.4, November 2011

\begin{tabular}{|c|c|c|c|}
\hline & & 70 & $\begin{array}{l}\text { Adaptive Median, Adaptive Median, Adaptive } \\
\text { Median, Adaptive Median, } 3 \text { x } 1 \text { vertical median }\end{array}$ \\
\hline & & 90 & $\begin{array}{l}\text { Adaptive Median, Adaptive Median, Adaptive } \\
\text { Median, Adaptive Median, } 3 \text { x } 1 \text { vertical median }\end{array}$ \\
\hline \multirow[t]{5}{*}{2} & \multirow[t]{5}{*}{ Knee } & 10 & Brightness value control with $\mathrm{s}=0$, Adaptive Median \\
\hline & & 30 & $\begin{array}{l}\text { Brightness value control with } \mathrm{s}=0 \text {, Adaptive Median, } \\
\qquad 3 \times 1 \text { vertical median }\end{array}$ \\
\hline & & $\mathbf{5 0}$ & $\begin{array}{l}\text { Adaptive Median, Adaptive Median, Adaptive } \\
\text { Median, Adaptive Median, } 3 \text { x } 1 \text { vertical median }\end{array}$ \\
\hline & & 70 & $\begin{array}{l}\text { Adaptive Median, Adaptive Median, Adaptive } \\
\text { Median, Adaptive Median, } 3 \text { x } 1 \text { vertical median }\end{array}$ \\
\hline & & 90 & 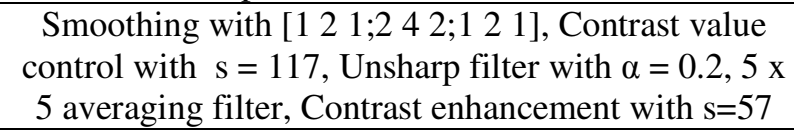 \\
\hline \multirow[t]{5}{*}{3} & \multirow{5}{*}{$\begin{array}{l}\text { Mammogra } \\
\text { m }\end{array}$} & $\mathbf{1 0}$ & Brightness value control with $\mathrm{s}=0$, Adaptive Median \\
\hline & & 30 & Brightness value control with $s=0$, Adaptive Median \\
\hline & & $\mathbf{5 0}$ & $\begin{array}{l}\text { Brightness value control with } \mathrm{s}=0 \text {, Adaptive Median, } \\
\qquad 3 \mathrm{x} 1 \text { vertical median }\end{array}$ \\
\hline & & 70 & $\begin{array}{l}\text { Adaptive Median, Adaptive Median, Adaptive } \\
\text { Median, Adaptive Median, } 3 \text { x } 1 \text { vertical median } \\
\end{array}$ \\
\hline & & 90 & $\begin{array}{l}\text { Adaptive Median, Adaptive Median, Adaptive } \\
\text { Median, Adaptive Median, } 3 \text { x } 1 \text { vertical median }\end{array}$ \\
\hline \multirow[t]{5}{*}{4} & \multirow[t]{5}{*}{ Head } & $\mathbf{1 0}$ & Brightness value control with $\mathrm{s}=0$, Adaptive Median \\
\hline & & 30 & Adaptive Median, Adaptive Median \\
\hline & & $\mathbf{5 0}$ & $\begin{array}{l}\text { Adaptive Median, Adaptive Median, Adaptive } \\
\text { Median, Adaptive Median, } 3 \text { x } 1 \text { vertical median }\end{array}$ \\
\hline & & 70 & $\begin{array}{c}\text { Adaptive Median, Unsharp filter with } \alpha=0.5,5 \times 5 \\
\text { averaging filter, Brightness value control with s=67, } \\
\text { Averaging with [2 } 4542 ; 491294 ; 51215125 ; 49 \\
1294 ; 24542]\end{array}$ \\
\hline & & 90 & 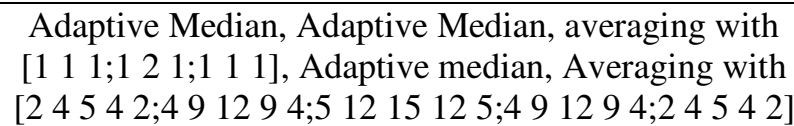 \\
\hline
\end{tabular}

\subsection{Performance Evaluation.}

The performance of FGA-F was compared with a single $5 \times 5$ median filter since a median filter (MF) is conventionally used for impulse noise removal [1]. FGA-F was also compared with variations of MF such as a $5 \times 5$ Weighted MF (WMF) [12] with a weight of [1 $111111 ; ; 1222$ 1;1 232 1; 1222 1;1 1111 1], a $5 \times 5$ Center Weighted MF (CWMF) [12]with a center weight of 3 and an Adaptive MF (AMF) [1] with a maximum window size of 7. All of these filters are standard methods for impulse noise removal.

Following are the metrics used for performance evaluation:

\section{Peak Signal to Noise Ratio (PSNR)}

PSNR value of a denoised image with respect to the original image is calculated as shown in (7). This value, represented in $\mathrm{dB}$, denotes the closeness of the denoised image to the original image. A high PSNR value for the denoised image shows its closeness to the original image.

$$
P S N R=10 * \log 10\left(255^{2} / M S E\right)
$$


The International Journal of Multimedia \& Its Applications (IJMA) Vol.3, No.4, November 2011

where MSE is the mean squared error.

\section{Tenengrad Measure}

Tenengrad measure indicates the amount of edge details present in an image. Higher the value, the more edge details present in the image. Tenengrad method is based on obtaining the gradient magnitude from the Sobel operator. It is calculated as shown in (8).

$$
T E N=\sum_{x=2}^{M-1} \sum_{y=2}^{N-1}(\nabla S(x, y))^{2} \text { for } \nabla \mathrm{S}(\mathrm{x}, \mathrm{y})>\mathrm{T}
$$

Where $\mathrm{T}$ is a discrimination threshold value and $\nabla S(x, y)$ is the Sobel gradient magnitude value. Here, $\mathrm{T}$ is taken to be zero. When the TEN for the denoised image $\mathrm{R}$ is close to the original image $\mathrm{O}$, it shows that the denoising process preserves the edge details in the image. TEN of $\mathrm{R}$ is less than TEN of $\mathrm{O}$ when the denoising process results in loss of edge details. TEN of $\mathrm{R}$ is greater than TEN of $\mathrm{O}$ when the denoising process creates false edge details.

\section{Image Quality Index (IQI)}

IQI [6] is designed by modelling any image distortion as a combination of three factors: lossof correlation, luminance distortion and contrast distortion. It is calculated as shown in (9). The value of IQI ranges from $[-1,1]$. A denoised image which is much similar to the original image has an IQI value close to one.

$$
I Q I=\operatorname{Corr}(O, R) * \operatorname{Lum}(O, R) * \operatorname{Cont}(O, R)
$$

\subsection{Experimental Analysis}

Table 4, 5, 6 and 7 shows the values of different metrics obtained for the brain, knee, mammogram and head images corrupted by noise model 1,2,3 and 4 respectively, denoised by the proposed FGA based composite filters (FGA - F) in table 3, MF, WMF, CWMF and AMF for the respective impulse noise levels. From the four tables, it can be seen that FGA based composite filters performs well on brain, knee and head images and exceptionally well on the mammogram image. The performance of FGA based composite filter is unsatisfactory only on high level noisy knee and head images. This indicates that FGA based composite filters do not perform well on high level model 2 and model 4 impulse noises. But, this is not a serious issue because medical images seldom gets affected by very high density noise due to the fact that very high quality equipments are used to capture medical images which may introduce only low density noise in the images. Thus, it can be safely said that FGA based composite filters can be applied on medical images for impulse noise removal.

The tables 4, 5, 6 and 7 show that for all noise models, using composite filters produced by FGA for impulse noise removal yields better results than using a single MF, WMF, CWMF or AMF, especially for high noise levels, as shown by the PSNR and IQI values. For low noise levels, the performance of FGA based composite filters is the same as AMF because FGA has found AMF to be the optimal filter for removing low level impulse noise. But, as the noise level increases, FGA based composite filters outperform AMF. The Tenengrad value for the original brain image is 659918, for knee image is 35432, for the mammogram image is 99602 and for the head image is 241774. TEN values produced by FGA based composite filters are much close to the original values than the other filters, thus indicating that FGA based composite filters preserves image details much more efficiently than the other filters. Altogether, the performance of the proposed FGA composite filters is much superior to the standard procedures for impulse noise removal from medical images. 
The International Journal of Multimedia \& Its Applications (IJMA) Vol.3, No.4, November 2011

Table 4. Comparing PSNR, Tenengrad measure and IQI obtained for Brain image corrupted with $10 \%, 30 \%, 50 \%, 70 \%$ and $90 \%$ model 1 impulse noise

\begin{tabular}{|c|c|c|c|c|c|c|}
\hline & Noise & MF & WMF & CWM & AMF & FGA - F \\
\hline \multirow[t]{5}{*}{$\operatorname{PSNR}(\mathrm{dB})$} & 10 & 19.008 & 18.11 & 18.04 & 26.434 & 26.4346 \\
\hline & 30 & 18.307 & 16.81 & 17.01 & 22.615 & 22.615 \\
\hline & 50 & 16.426 & 14.86 & 15.13 & 19.355 & 19.5416 \\
\hline & 70 & 11.485 & 12.47 & 12.70 & 15.554 & 17.0378 \\
\hline & 90 & 6.3801 & 9.097 & 9.413 & 8.5209 & 12.644 \\
\hline \multirow{5}{*}{ IQI } & 10 & 0.5998 & 0.561 & 0.555 & 0.9221 & 0.92207 \\
\hline & 30 & 0.5712 & 0.484 & 0.488 & 0.8693 & 0.86933 \\
\hline & 50 & 0.4784 & 0.374 & 0.385 & 0.7705 & 0.75316 \\
\hline & 70 & 0.2233 & 0.245 & 0.253 & 0.572 & 0.5616 \\
\hline & 90 & 0.049 & 0.097 & 0.098 & 0.128 & 0.3205 \\
\hline TEN & 10 & 40804 & 38563 & 37101 & 57757 & 577570 \\
\hline \multirow{4}{*}{$\begin{array}{l}\text { Original Brain image TEN }= \\
659918\end{array}$} & 30 & 41370 & 29365 & 30942 & 52123 & 521239 \\
\hline & 50 & 30823 & 30731 & 30271 & 52217 & 469164 \\
\hline & 70 & 17308 & 16970 & 38938 & 17532 & 955180 \\
\hline & 90 & 19715 & 39082 & 46327 & 76729 & 451548 \\
\hline
\end{tabular}

Table 5. Comparing PSNR, Tenengrad measure and IQI obtained for Knee image corrupted with $10 \%, 30 \%, 50 \%, 70 \%$ and $90 \%$ model 2 impulse noise

\begin{tabular}{|c|c|c|c|c|c|c|}
\hline & Noise \% & MF & WMF & CWMF & AMF & FGA - F \\
\hline \multirow[t]{5}{*}{ PSNR(dB) } & 10 & 26.8601 & 26.6929 & 26.568 & 34.6784 & 34.6784 \\
\hline & 30 & 20.8782 & 19.1774 & 19.8576 & 26.8958 & 27.6117 \\
\hline & 50 & 9.0075 & 11.0772 & 12.2495 & 13.5178 & 20.7197 \\
\hline & 70 & 8.3766 & 6.9575 & 6.8707 & 10.6353 & 13.2657 \\
\hline & 90 & 2.7231 & 2.6079 & 2.6077 & 2.8284 & 7.5605 \\
\hline \multirow[t]{5}{*}{ IQI } & 10 & 0.4967 & 0.4739 & 0.4715 & 0.8838 & 0.88385 \\
\hline & 30 & 0.4206 & 0.3539 & 0.365 & 0.7889 & 0.7247 \\
\hline & 50 & 0.1913 & 0.1548 & 0.1751 & 0.4363 & 0.45717 \\
\hline & 70 & 0.0656 & 0.0196 & 0.0218 & 0.2311 & 0.25334 \\
\hline & 90 & 0.0033 & 0 & 0 & 0.0168 & 0.021583 \\
\hline TEN & 10 & 131860 & 174375 & 114729 & 39645 & 39645 \\
\hline \multirow{4}{*}{$\begin{array}{l}\text { Original Knee image } \\
\quad \text { TEN }=35432\end{array}$} & 30 & 186901 & 128462 & 128950 & 53292 & 58570 \\
\hline & 50 & 934012 & 1542267 & 1542267 & 100744 & 112133 \\
\hline & 70 & 0 & 0 & 0 & 754778 & 6840 \\
\hline & 90 & 1755675 & 1755675 & 1755675 & 2017926 & 209502 \\
\hline
\end{tabular}


The International Journal of Multimedia \& Its Applications (IJMA) Vol.3, No.4, November 2011

Table 6. Comparing PSNR, Tenengrad measure and IQI obtained for Mammogram image corrupted with $10 \%, 30 \%, 50 \%, 70 \%$ and $90 \%$ model 3 impulse noise

\begin{tabular}{|c|c|c|c|c|c|c|}
\hline & Noise \% & MF & WMF & CWMF & AMF & FGA - F \\
\hline \multirow[t]{5}{*}{$\operatorname{PSNR}(\mathrm{dB})$} & 10 & 30.5777 & 31.083 & 31.0437 & 39.047 & 39.047 \\
\hline & 30 & 27.8437 & 29.3175 & 29.5848 & 35.586 & 35.586 \\
\hline & 50 & 22.7853 & 26.5076 & 26.735 & 31.6955 & 31.8552 \\
\hline & 70 & 13.4704 & 20.964 & 21.7738 & 21.4689 & 29.0352 \\
\hline & 90 & 6.5692 & 14.6863 & 15.3842 & 9.4986 & 18.0228 \\
\hline \multirow[t]{5}{*}{ IQI } & 10 & 0.7118 & 0.6734 & 0.6701 & 0.9277 & 0.92772 \\
\hline & 30 & 0.5998 & 0.5787 & 0.5868 & 0.6694 & 0.66938 \\
\hline & 50 & 0.3438 & 0.2594 & 0.2832 & 0.5686 & 0.54747 \\
\hline & 70 & 0.091 & 0.1287 & 0.1393 & 0.3935 & 0.3966 \\
\hline & 90 & 0.0068 & 0.0415 & 0.0514 & 0.033 & 0.19914 \\
\hline TEN & 10 & 120200 & 123791 & 124954 & 101535 & 101535 \\
\hline \multirow{4}{*}{$\begin{array}{l}\text { Original Mammogram } \\
\text { image TEN }=99602\end{array}$} & 30 & 107248 & 130917 & 123069 & 99116 & 99116 \\
\hline & 50 & 107533 & 130304 & 131748 & 83605 & 85452 \\
\hline & 70 & 64094 & 142738 & 138490 & 58524 & 72987 \\
\hline & 90 & 2639924 & 999429 & 159523 & 152245 & 147802 \\
\hline
\end{tabular}

Table 7. Comparing PSNR, Tenengrad measure and IQI obtained for Head image corrupted with $10 \%, 30 \%, 50 \%, 70 \%$ and $90 \%$ model 4 impulse noise

\begin{tabular}{|c|c|c|c|c|c|c|}
\hline & Noise \% & MF & WMF & CWMF & AMF & FGA - F \\
\hline \multirow[t]{5}{*}{$\operatorname{PSNR}(\mathrm{dB})$} & 10 & 19.4353 & 19.0565 & 19.0878 & 27.3558 & 27.3558 \\
\hline & 30 & 18.2185 & 17.0826 & 17.3723 & 22.5272 & 22.5322 \\
\hline & 50 & 14.5257 & 13.5971 & 14.1913 & 18.57 & 19.6345 \\
\hline & 70 & 7.3828 & 7.1537 & 7.1537 & 8.2383 & 9.8193 \\
\hline & 90 & 7.8853 & 7.1538 & 7.1809 & 9.4845 & 10.8876 \\
\hline \multirow[t]{5}{*}{ IQI } & 10 & 0.7377 & 0.7073 & 0.7081 & 0.9347 & 0.9347 \\
\hline & 30 & 0.6707 & 0.5989 & 0.6118 & 0.758 & 0.84956 \\
\hline & 50 & 0.4354 & 0.3214 & 0.3173 & 0.6376 & 0.61164 \\
\hline & 70 & 0.0079 & 0 & 0 & 0.0877 & 0.14673 \\
\hline & 90 & 0.0594 & 0.0013 & 0.0012 & 0.1434 & 0.17321 \\
\hline TEN & 10 & 32868 & 57974 & 54997 & 266421 & 266421 \\
\hline \multirow{4}{*}{$\begin{array}{l}\text { Original Head image } \\
\text { TEN }=241774\end{array}$} & 30 & 88469 & 68737 & 72446 & 190963 & 193268 \\
\hline & 50 & 95423 & 230413 & 287301 & 178644 & 76058 \\
\hline & 70 & 108 & 108 & 108 & 5334 & 53559 \\
\hline & 90 & 2084170 & 432 & 432 & 1010429 & 39997 \\
\hline
\end{tabular}

Figure 3 shows the results produced by MF, WMF, CWMF, AMF and the proposed FGA based composite filters for the head image with $10 \%$ model 4 impulse noise with interval 4 , mammogram image with 30\% model 3 impulse noise with interval 6, knee with 50\% model 2 impulse noise and brain image with $70 \%$ model 1 impulse noise. The MF can completely denoise 
The International Journal of Multimedia \& Its Applications (IJMA) Vol.3, No.4, November 2011

images with low noise levels, but it performs poorly on images with high noise level. The output images produced by WMF and CWMF are dull in appearance for low noise levels, and have a smeared appearance for high noise levels, thereby resulting in a considerable loss of image details. AMF efficiently removes low noise levels from the images. But, for high noise levels, the output images are not completely denoised, and there is a considerable loss of image details, as can be seen in the knee and brain images.

It can be seen that for all noise levels, the outputs produced by FGA based composite filters are completely denoised with considerable image detail preservation. This is clear from the fact that the proposed filter, along with noise removal, retains the high density white spots in the denoised brain image which was present in the original brain image, even when the input noise level is high (70\%). Thus, it can be safely said that FGA based composite filters can be used for impulse noise removal with a minimal loss of significant details from the image. This makes the FGA based composite filters suitable for use in medical image analysis to remove impulse noise from medical images.

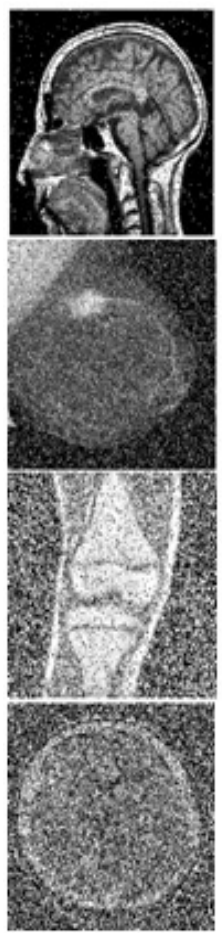

(a)

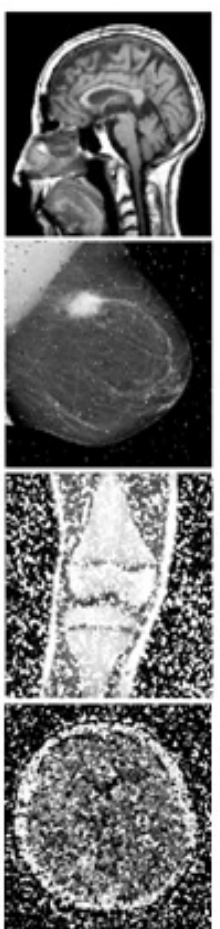

(b)

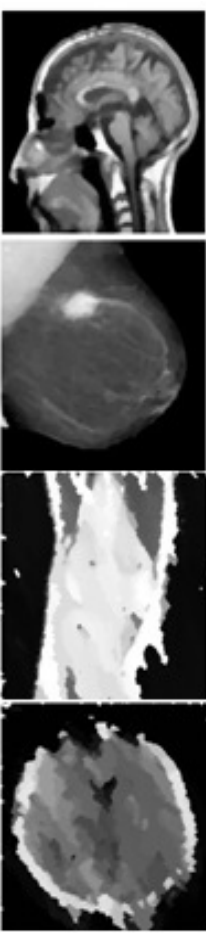

(c)

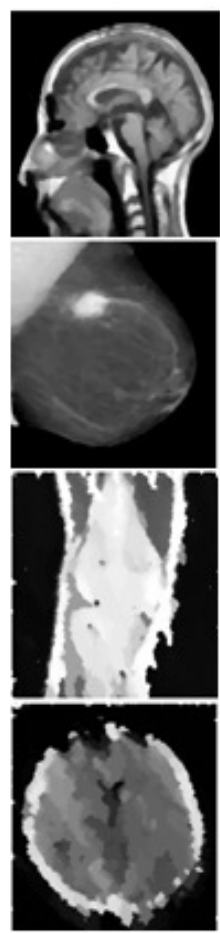

(d)

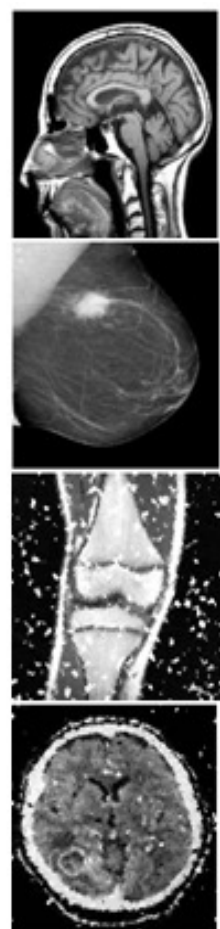

(e)

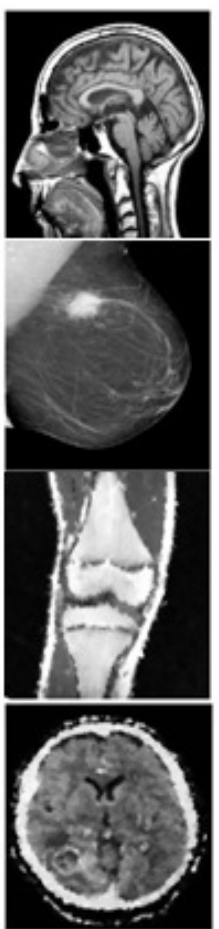

(f)

Figure 3. Column (a): Head with $10 \%$ model 4 impulse noise with noise intensity interval 4, Mammogram with $30 \%$ model 3 impulse noise with noise intensity interval of 6, Knee with 50\% model 2 impulse noise and Brain with 70\% model model 1 impulse noise. Column (b), (c), (d),

(e) and (f): Outputs produced by MF, WMF, CWMF, AMF and the proposed FGA based composite filters respectively

\section{Conclusions}

Medical images are susceptible to impulse noise, which results in inaccurate analysis that can prove disastrous. Median filters and its variants are commonly used for impulse noise removal, but they result in loss of image details. Also, for high noise levels, they are not sufficient in completely removing the noise. In this paper, FGA is used to determine the optimal composite 
The International Journal of Multimedia \& Its Applications (IJMA) Vol.3, No.4, November 2011

filters for removing different levels of impulse noise from medical images without using deep knowledge about noise factors. Here, a FRB is used to adaptively change the crossover probability of GA. Experiments conducted show that the proposed method is much better than the standard procedures for removing impulse noise from medical images along with image detail preservation. As future work, the proposed method can be used in applications such as impulse noise removal from satellite images.

\section{ACKNOWLEDGEMENTS}

The authors would like to thank each and everyone who have helped in the creation of this paper.

\section{REFERENCES}

[1] Gonzalez, R. and Woods, R. (1992) Digital Image Processing, Addison Wesley, Reading, MA.

[2] Goldberg, G (1989) Genetic Algorithm in Search, Optimization and Learning, Addison Wesley.

[3] Jin Hyuk Hong, Sung Bae Cho and Ung Keun Cho (2009) "A Novel Evolutionary Method to Image Enhancement Filter Design: Method and Applications", IEEE Transactions on Systems, Man and Cybernetics - Part B, Cybernetics, Vol. 39, No. 6, pp. 1446-1457.

[4] Jin Hyuk Hong, Sung Bae Cho and Ung Keun Cho (2006) "Image Enhancement for Impulsive Noise Reduction”, Huang, D.S., Li, K., Irwin, G.W., (eds.) ICIC 2006. LNCS, Vol. 4113, pp. 678--683. Springer, Heidelberg.

[5] Herrera, F. and Lozano, M (1996) "Adaptive Genetic Algorithms based on Fuzzy Techniques", Proceedings of the Sixth International Conference on Information Processing and Management Uncertainty in Knowledge Based Systems, IEEE, pp. 775-780.

[6] Wang, Z. and Bovik, A.C (2002) “A universal image quality index”, IEEE Transactions on Signal Processing Letters, 9(3), pp. 81-84.

[7] Ross, T. J (1995) Fuzzy Logic with Engineering Applications, McGraw Hill.

[8] Herrera, F. and Lozano, M (2001) "Adaptive Genetic Operators Based on Co evolution with Fuzzy Behaviours", IEEE Transactions on Evolutionary Computation, Vol. 5, No. 2, pp. 149-165.

[9] Lee, M. A. and Hideyuki Takagi (1993) "Dynamic Control of Genetic Algorithms using Fuzzy Logic Techniques", Proceedings of Fifth International Conference on Genetic Algorithms, Urbana Champaign, IL, pp. 76-83.

[10] Cordon, O., Herrera, F., Hoffmann, F. and Magdalena, L (2001) "Genetic Fuzzy Systems Evolutionary Tuning and Learning of Fuzzy Knowledge Bases", Advances in Fuzzy Systems Applications and Theory, Vol. 19, World Scientific Publishing Co. Pte. Ltd.

[11] Madhu S. Nair and G. Raju (2010) “A new fuzzy-based decision algorithm for high-density impulse noise removal”, Springer-Verlag, London Limited. Signal Image and Video processing. DOI 10.1007/s11760-010-0186-4.

[12] Sung JeaKo and Yong Hoon Lee (1991) "Center Weighted Median Filters and their application to Image Enhancement”, IEEE Transactions on Circuits and Systems, Vol 38, No 9. 
The International Journal of Multimedia \& Its Applications (IJMA) Vol.3, No.4, November 2011

\section{Authors}

Anisha K K obtained her B.Tech in Computer Science and Engineering from Government Engineering College, Sreekrishnapuram, Kerala, India under University of Calicut, Kerala. She is currently pursuing her M.Tech degree in Digital Image Computing from University of Kerala, Kariavattom, Thiruvananthapuram, Kerala, India.

Dr. M. Wilscy received her B.Sc (Engg.) degree in Electrical Engineering from Kerala University, ME in Automation from School of Automation, Indian Institute of Science, Bangalore and Ph.D from Indian Institute of Technology, Madras. She is currently the Head of the Computer Science department of University of Kerala, Kariavattom, Thiruvananthapuram, Kerala, India. She has more than 30 years of teaching experience and her current research interests include applying intelligent techniques to Digital Image Processing. She is a Fellow of the Institution of Engineers India, a Member of IEEE, and member of Indian Society of Technical Education 